\title{
Precision of coherence analysis to detect cerebral autoregulation by near-infrared spectroscopy in preterm infants
}

Gitte Holst Hahn

Copenhagen University Hospital - Rigshospitalet Department of Neonatology

Blegdamsvej 9

Copenhagen, 2100 Denmark

\section{Karl Bang Christensen}

University of Copenhagen

Department of Biostatistics

Copenhagen, 2100 Denmark

\section{Terence S. Leung}

University College London

Department of Medical Physics and Bioengineering Gower Street

London, WC1E 6BT United Kingdom

\section{Gorm Greisen}

Copenhagen University Hospital - Rigshospitalet Department of Neonatology

Blegdamsvej 9

Copenhagen, 2100 Denmark

\begin{abstract}
Coherence between spontaneous fluctuations in arterial blood pressure (ABP) and the cerebral near-infrared spectroscopy signal can detect cerebral autoregulation. Because reliable measurement depends on signals with high signal-to-noise ratio, we hypothesized that coherence is more precisely determined when fluctuations in $\mathrm{ABP}$ are large rather than small. Therefore, we investigated whether adjusting for variability in $\mathrm{ABP}$ (variability ${ }_{\mathrm{ABP}}$ ) improves precision. We examined the impact of variability ${ }_{A B P}$ within the power spectrum in each measurement and between repeated measurements in preterm infants. We also examined total monitoring time required to discriminate among infants with a simulation study. We studied 22 preterm infants $(G A<30)$ yielding 21510 -min measurements. Surprisingly, adjusting for variability ${ }_{A B P}$ within the power spectrum did not improve the precision. However, adjusting for the variability ${ }_{\mathrm{ABP}}$ among repeated measurements (i.e., weighting measurements with high variability $_{A B P}$ in favor of those with low) improved the precision. The evidence of drift in individual infants was weak. Minimum monitoring time needed to discriminate among infants was $1.3-3.7 \mathrm{~h}$. Coherence analysis in low frequencies $(0.04-0.1 \mathrm{~Hz})$ had higher precision and statistically more power than in very low frequencies $(0.003-0.04 \mathrm{~Hz})$. In conclusion, a reliable detection of cerebral autoregulation takes hours and the precision is improved by adjusting for variability $_{A B P}$ between repeated measurements. $\odot 2010$ Society of PhotoOptical Instrumentation Engineers. [DOI: 10.1117/1.3426323]
\end{abstract}

Keywords: spectroscopy; coherence; Fourier transforms; medicine.

Paper 09256RR received Jun. 19, 2009; revised manuscript received Mar. 3, 2010; accepted for publication Mar. 9, 2010; published online May 12, 2010.

\section{Introduction}

Near-infrared spectroscopy (NIRS) has been used in clinical research to study cerebral intravascular oxygenation in newborns for more than 20 years. ${ }^{1}$ Recently, NIRS has also been introduced to detect cerebral autoregulation. Cerebral autoregulation is a physiological mechanism that protects the brain against a fluctuating arterial blood pressure (ABP) by maintaining an approximately constant level of cerebral blood flow. ${ }^{2}$ This is achieved through dilatation or constriction of cerebral blood vessels. The assessment of cerebral autoregulation is based on analysis of coherence between changes in cerebral intravascular oxygenation and changes in ABP. Using spontaneous fluctuations in ABP, this method offers a potential clinical tool to monitor cerebral autoregulation noninvasively and continuously in premature infants. Potentially, this can guide therapeutic interventions and therefore prevent brain damage. $^{3}$

Address all correspondence to: Gitte Holst Hahn, Copenhagen University Hospital - Rigshospitalet, Department of Neonatology, Blegdamsvej 9, Copenhagen, 2100 Denmark. Tel.: 45-35454338; Fax: 45-35455025; E-mail: gitte.holst.hahn@rh.regionh.dk
Coherence is a frequency domain estimate of linear correlation. It estimates the degree to which the variance in cerebral intravascular oxygenation at a certain frequency can be explained by the variance of the $\mathrm{ABP}$ at the same frequency. Coherence ranges from 0 to 1 , with 1 indicating perfect correlation and 0 complete lack of correlation. High coherence between $\mathrm{ABP}$ and cerebral intravascular oxygenation indicates imperfect cerebral autoregulation, while low coherence indicates effective autoregulation (Fig. 1). ${ }^{4}$ Coherence provides a qualitative but not a quantitative measure of cerebral autoregulation. Quantitative assessment of autoregulation (i.e., the magnitude of pressure passivity) requires estimation of the operational gain or amplification factor between the two signals. However, quantification of operational gain is only reasonable if coherence is significant. Thus, an imprecise estimate of coherence makes analysis of gain inappropriate. Consequently, our study concentrates exclusively on optimizing the precision of coherence analysis to detect cerebral autoregulation.

A reliable detection of cerebral autoregulation requires a certain challenge to the autoregulatory system (i.e., a certain

$1083-3668 / 2010 / 15(3) / 037002 / 10 / \$ 25.00$ C 2010 SPIE 

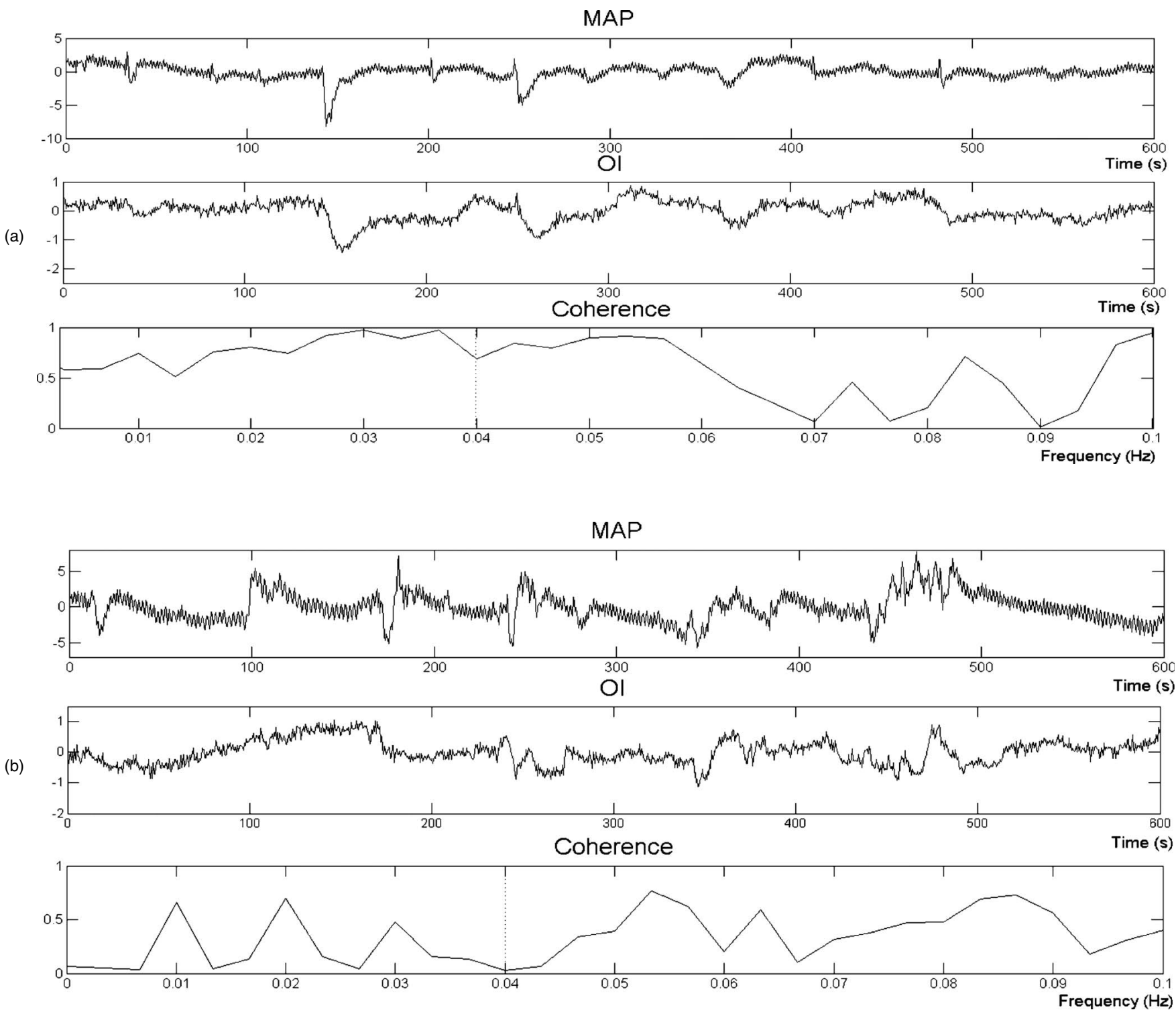

Fig. 1 Example of simultaneous changes in the detrended signals of MAP and OI and their coherence-spectrum illustrating (a) high and (b) low coherence. The dotted line represents the shift from VLF to LF range.

amount of ABP changes). ${ }^{4-6}$ In vulnerable patients such as premature infants, however, use of spontaneous ABP changes is preferable to induced changes. ABP with small changes has low values in its power spectral density (PSD). Detecting cerebral autoregulation on the basis of such a signal could be misleading and falsely indicate a low coherence and, therefore, effective cerebral autoregulation, whereas the variability of cerebral oxygenation is in fact dominated by "noise," e.g., (i) physiological noise (such as variability of arterial content of carbon dioxide, arterial saturation or cerebral oxygen uptake) or (ii) instrumental noise (due to limitations of the optics, electronics or movements).

Previous reports using coherence to detect cerebral autoregulation have not adjusted for variability in $\mathrm{ABP}^{7-9}$ This analytical approach has shown a lack of stability, with cerebral autoregulation that fluctuates (i.e., that comes and goes over short periods of time). ${ }^{8}$ Thus, we set out to investigate whether adjusting for the magnitude of ABP changes would lead to a more precise detection of cerebral autoregulation.

The hypothesis of our study was that coherence would be more precisely determined when ABP changes were large rather than small. In general, a reliable test of a functional relation requires that the stimulus itself exceeds the noise level of the measurements (i.e., a high signal-to-noise ratio). Hence, in the present study we examined the effect of ABP changes at specific frequencies [i.e., the effect of the shape of the power spectrum in each measurement (intra-measurement adjustment)]. We also examined the effect of ABP sometimes varying greatly and sometimes varying a little, by performing a weighted analysis where, out of several measurements in each infant, we weighted those with large ABP changes higher than those with small (intermeasurement adjustment). 


\section{Materials and Methods}

\subsection{Participants}

We studied 22 premature infants with a gestational age of $<32$ weeks with an indwelling arterial catheter, which had been inserted for clinical reasons at birth. The study was carried out at the Neonatal Care Unit, Copenhagen University Hospital, Rigshospitalet, Copenhagen. The study was conducted in parallel with an ongoing study on the link between fetal inflammation and cerebral autoregulation, and hence, only children born at Rigshospitalet were eligible. Infants with major malformations or diagnosis of brain injury at recruitment were excluded. We aimed to examine infants within the first $24 \mathrm{~h}$ after birth. Median age at examination was 17.4 (4.0-43.5) h. Clinical data were obtained from the infants' medical records. The latest blood gas value taken before the examination was also recorded [median time interval between blood sample and examination: $2.0(0-6.4) \mathrm{h}]$ : Demographic and clinical data of the infants are summarized in Table 1 . The Danish Local Ethical Committee approved the study (Journal No. H-A-2007-0109), and written informed parental consent was obtained in all cases.

\subsection{Continuous NIRS and Arterial Blood Pressure Recording}

NIRS data were recorded using a NIRO-300 spectrophotometer (Hamamatsu Photonics, Hamamatsu City, Japan). The probes were fixed in a nontransparent, soft probe holder and secured to the frontotemporal or frontoparietal region of the head with a flexible bandage. The interoptode distance was $4 \mathrm{~cm}$. Because the skin of premature infants is very thin and vulnerable, no adhesive tape was used. Changes in the relative concentrations of oxygenated and deoxygenated haemoglobin were recorded and used to calculate the oxygenation index (OI). OI is the difference between oxygenated and deoxygenated haemoglobin divided by a factor of 2. Changes in OI have been validated to represent changes in cerebral blood flow in several animal studies. ${ }^{10-12}$

$\mathrm{ABP}$ waveforms were measured with a pressure transducer connected to the indwelling arterial catheter. Before the mean ABP (MAP) was calculated, the ABP data were filtered by a first-order low-pass filter with a cutoff frequency of $0.3 \mathrm{~Hz}$.

NIRS and MAP data were sampled simultaneously and synchronized with arterial saturation (assessed by pulse oximetry) at $2 \mathrm{~Hz}$ by an analog-to-digital converter, and stored on a laptop for further analysis. Using in-house written software (Labview) recording was automatically stopped if changes in arterial saturation exceeded 5\%. The infants were clinically stable during measurements. One investigator (G.H.H.) observed the infants and the NIRS instrument continuously during the measurements and interrupted the recording if movement artefacts caused a baseline shift (e.g., radiographs and manipulation of the infant due to suctioning, etc.) or if there was loss of the ABP signal due to blood-gas sampling. To eliminate episodes of artifacts, the data were divided into 10-min measurements of uninterrupted recordings.

\subsection{Frequency Analysis}

Transfer function analysis of coherence between continuous measurements of OI and MAP were performed in two fre-
Table 1 Demographic data.

No. of infants

Sex $[\mathrm{no}]$

Gestational age [weeks]

Birth weight $[\mathrm{g}]$

Apgar at $5 \mathrm{~min}$

Umbilical cord $\mathrm{pH}$

\section{Circulatory support in the first day of life}

$\begin{array}{ll}\text { Inotropics }[\text { no }(\%)] & 2(9 \%) \\ \text { Volume treatment [no (\%)] } & 6(27 \%) \\ \text { Intropics or volume treatment [no (\%)] } & 6(27 \%)\end{array}$

\section{Clinical state at measurement}

$\begin{array}{lc}\text { Postnatal age }[\mathrm{h}] & 17.4[4.0-43.5] \\ \text { Inotropics [no (\%)] } & 2(9 \%) \\ \text { Nasal-CPAP [no (\%)] } & 20(91 \%) \\ \text { Mechanical ventilation [no (\%)] } & 2(9 \%) \\ \text { Inspiratory oxygen concentration [\%] } & 25[21-40] \\ \text { Arterial oxygen saturation [\%] } & 95[85-99] \\ \mathrm{P}_{\mathrm{a}} \mathrm{CO}_{2}[\mathrm{kPa}] & 5.8[4.4-8.2] \\ \mathrm{Pa}_{\mathrm{a}} \mathrm{O}_{2}[\mathrm{kPa}] & 8.0[4.0-14.0] \\ \mathrm{Haemoglobin}[\mathrm{mM}] & 9.2[5.5-11.9] \\ \mathrm{MAP}[\mathrm{mm} \mathrm{Hg}] & 35[26-42]\end{array}$

Later outcome

Intraventricular haemorrhage (all)

[no (\%)]

Intraventricular haemorrhage (Gr. III/IV)

[no (\%)]

Cystic perventricular leucomalacia

[no $(\%)]$

Neonatal mortality [no (\%)]

$2(9 \%)$ males/12 females

$5[24.1-29.4]$

$10[6-10]$

$7.32[7.03-7.45]$
Median and [range] unless otherwise stated.

quency ranges. The very low-frequency (VLF) range (0.003-0.04 Hz, corresponding to a cycle length of $25-300 \mathrm{~s})$ and the low-frequency (LF) range $(0.04-0.1 \mathrm{~Hz}$, corresponding to a cycle length of $10-25 \mathrm{~s}$ ). Each 10-min measurement was subdivided into three segments of $5 \mathrm{~min}$ with $50 \%$ overlap and a Hanning window was applied to minimize spectral leakage. Both time signals were then detrended and transformed into PSDs by Fourier transform (Matlab, MathWorks, Natick, Massachusetts). 
(a)

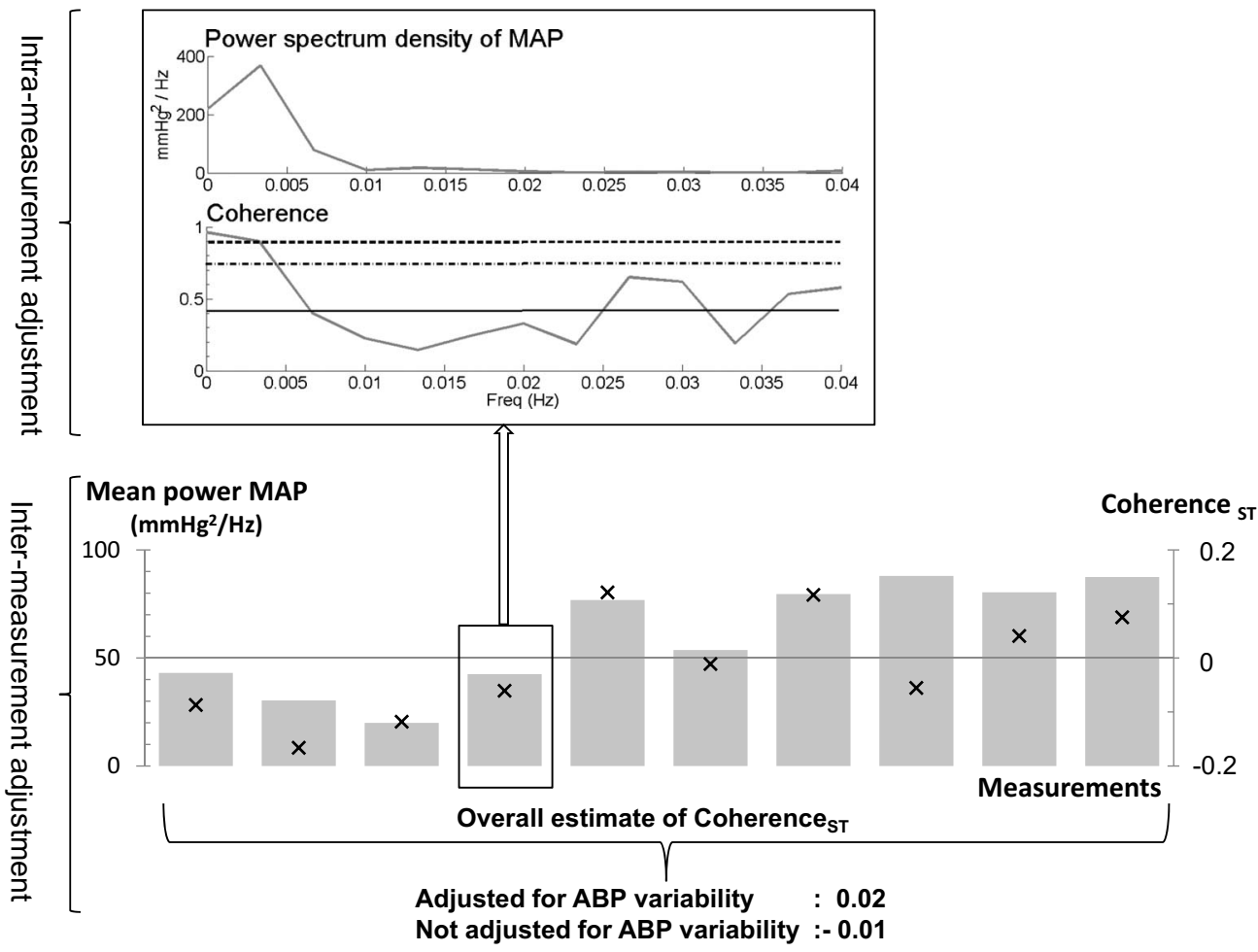

Fig. 2 Example of ten 10-min measurements in one of the infants (infant number 20) in the VLF range. (b) Illustrates the effect of adjusting for ABP variability among repeated measurements (intermeasurement adjustment). In this example, the measurements with high ABP variability (i.e., mean power of MAP, illustrated with bars) have the highest Coherence ${ }_{S T}$ (cross), and hence, the adjusted overall estimate of Coherence is $_{\text {igher }}$ than the unadjusted. In this case, the estimate even changes from being statistically insignificant to statistically significant $\left(\right.$ Coherence $\left.{ }_{S T} \geqslant 0\right)$. (a) Illustrates the effect of adjusting for ABP variability within measurement number 4 (intrameasurement adjustment). Mean coherence (Coh mean ) [solid line], intrameasurement ABP weighted mean coherence $\left(\mathrm{Coh}_{\text {MAPwmean }}\right)$ [dashed-doted line], and coherence at the frequency with maximum $\mathrm{PSD}_{\text {MAP }}\left(\mathrm{Coh}_{\text {MAPmax }}\right)$ [dashed line] are shown.

\subsection{Adjustment for Variability in $A B P$}

We adjusted for variability in ABP in two steps: $(i)$ within each measurement (intrameasurement adjustment) and (ii) between repeated measurements (intermeasurement adjustment) (Fig. 2). For intrameasurement adjustment, we calculated coherence in three different ways, as follows:

1. Mean value of the coherence spectrum $\left(\mathrm{Coh}_{\text {mean }}\right)$, i.e., no adjustment for variability in ABP

$$
\mathrm{Coh}_{\text {mean }}=\frac{1}{N} \sum_{i=1}^{N} \operatorname{Coh}\left(f_{i}\right),
$$

where $N$ is the number of PSD values within the predefined frequency range and $f_{i}$ is the $i$ 'th frequency within the frequency range.

2. Intrameasurement $\mathrm{ABP}$ weighted mean value according to the distribution of $\mathrm{PSD}_{\mathrm{MAP}}\left(\mathrm{Coh}_{\mathrm{MAPwmean}}\right)$, thereby attaching most weight to the frequencies with highest variability in $\mathrm{ABP}$,

$$
\operatorname{Coh}_{\text {MAPwmean }}=\sum_{i=1}^{N} w_{i} \operatorname{Coh}\left(f_{i}\right),
$$

where $w_{i}=\operatorname{PSD}_{\mathrm{MAP}}\left(f_{i}\right) / \sum_{i=1}^{N} \operatorname{PSD}_{\mathrm{MAP}}\left(f_{i}\right)$ and $\sum_{i=1}^{N} w_{i}=1$.

3. Point measurement at the frequency with maximum $\operatorname{PSD}_{\text {MAP }}\left(\right.$ Coh $\left._{\text {MAPmax }}\right)$,

$$
\operatorname{Coh}_{\text {MAP max }}=\operatorname{Coh}\left(f_{\max \text { MAP }}\right) \text {, }
$$

where $f_{\text {maxMAP }}$ corresponds to the frequency at which $\mathrm{PSD}_{\mathrm{MAP}}$ has the maximum value within the predefined frequency range.

For intermeasurement adjustment, we adjusted each infant's overall estimate of coherence for the varying degree of variability in ABP between repeated measurements. This was performed by a weighted analysis. We calculated a weighting factor for each measurement as the proportion of mean $\mathrm{PSD}_{\mathrm{MAP}}$ in the measurement concerned compared to the overall sum of mean PSD $_{\text {MAP }}$ in each infant. This factor, reaching the sum of 1 in each infant, was used to weight each repeated measurement. The weighted model is referred to as "intermeasurement ABP weighting."

\subsection{Threshold of Significant Coherence}

We estimated the $95 \%$ confidence limit for each coherence measurement mentioned above using Monte Carlo simulations. This confidence limit was then used as a threshold (Threshold $\left.\mathrm{COH}_{\mathrm{CO}}\right)$ to determine whether a measurement is coherent or not (i.e., coherent if the coherence measurement is above Threshold $\mathrm{COH}_{\mathrm{H}}$ ). In the Monte Carlo simulations, OI and MAP signals were both reshuffled randomly to obtain uncorrelated signals with signal variances that were the same as the original signal. The same procedure was repeated 10,000 
times, and 10,000 values resulted for each coherence measurement. These 10,000 values were then sorted in ascending order, and the $95 \%$ percentile was taken as the $95 \%$ confi-

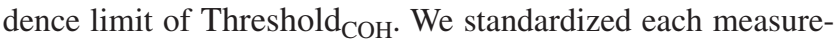
ment of coherence by subtracting Threshold $\mathrm{COH}_{\mathrm{C}}$ from it yielding standardized coherence (Coherence ${ }_{\mathrm{ST}}$ ). Coherence $_{\mathrm{ST}} \geqslant 0$ indicates imperfect cerebral autoregulation.

\subsection{Measure of Precision}

We applied different analytical approaches to the same data and compared their precision in terms of repeatability between repeated measurements. We hypothesized that cerebral autoregulation stayed stable between two successive 10-min measurements, and hence, that a high repeatability reflects a high signal-to-noise ratio (i.e., a low amount of measurement error). Statistically, we looked for the analytical approach with the best discrimination among infants based on their level of coherence. This means that we looked for the analytical approach with the highest variability in level of coherence among infants (i.e., a large range of estimates among infants) that at the same time had a small range of variation between repeated measurements in the same infant [i.e., a small standard error $(\mathrm{SE})]$. These conditions are combined in the $P$ value. Hence, we chose to favor the analytical approach with the most significant difference in level of coherence among our infants.

\subsection{Statistical Methods}

Averages of coherence, Coherence $\mathrm{ST}_{\mathrm{ST}}$, ThresholdCOH, and variability in MAP were calculated for each infant, and from these averages, we calculated a grand mean. Variability in MAP in each measurement was calculated as the square root of mean $\mathrm{PSD}_{\mathrm{MAP}}$ in each frequency range. To estimate the variability in mean PSD $_{\text {MAP }}$ between measurements, we calculated each infant's coefficient of variation.

One-way analysis of variance for repeated measurements with spatial power as covariance structure was used to describe each infants overall estimate of Coherence ST ("proc mixed" in SAS 9.1, SAS Institute). This covariance structure takes the nonequidistant nature of the measurements due to artifacts, blood-gas sampling, or changes in arterial saturation exceeding 5\% into account. We used two models. Model 1 used infant identification as the only covariate, allowing each

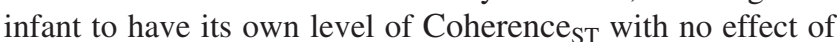
time. Model 2 added time and interaction between time and infant identification, thereby allowing each infant to have an individual level and trend of Coherence ST $_{\text {. We used model } 2}$ to identify a possible drifting nature of cerebral autoregulation. We used model 1 to calculate each infant's overall estimate of coherence and to divide the infants into the two following groups: effective autoregulation (i.e., Coherence ${ }_{\mathrm{ST}}$ $<0$ ) and imperfect autoregulation (i.e., Coherence ST $_{2} \geqslant 0$ ). An independent-samples T-test was conducted to compare gestational age (GA) and postnatal age for infants in these two groups. A nonparametric Fisher's exact test was used to test if clinical state (use of inotropics or mechanical ventilation), incidence of intraventricular haemorrhage (IVH) grade 3-4, or death in the first month of life differed between these groups (SPSS statistics 17.0). $P<0.05$ was considered statistically significant.
In order to estimate the minimum monitoring time needed to reach a robust estimate of cerebral autoregulation, we examined statistical power of $\mathrm{Coh}_{\text {mean }}$, $\mathrm{Coh}_{\mathrm{MAP} w m e a n}$, and $\mathrm{Coh}_{\text {MAPmax }}$ by means of a simulation study. Using estimated

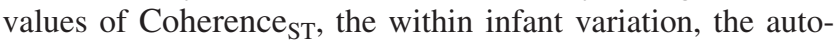
correlation, and the residual variation from model 1 with an autoregressive structure, we generated data sets with 22 infants for each of the three measurements of coherence. The number of measurements varied between 5 and 35. Statistical power according to number of measurements was then estimated by the empirical rejection rate in the 1000 simulated data sets.

\section{Results}

\subsection{General Features of Blood Pressure, Coherence, and Threshold of Coherence}

We monitored 22 infants continuously with a median duration of $2.1(1.2-3.7) \mathrm{h}$ yielding 215 measurements of $10 \mathrm{~min}$ [median: 10, (4-17)]. Grand mean variability in MAP was $4.8 \mathrm{~mm} \mathrm{Hg}$ (SD: 1.5) in VLF and $1.8 \mathrm{~mm} \mathrm{Hg}$ (SD: 0.7) in LF. Mean coefficient of variation in mean PSD $_{\mathrm{MAP}}$ between measurements was 62\% (SD: $21 \%$ ) and 50\% (SD: $21 \%$ ) for VLF and LF, respectively ( $P$ : 0.06$)$.

$\mathrm{Coh}_{\text {MAPmax }}$ had the highest Threshold ${ }_{\mathrm{COH}}$ in both frequency bands [0.80 (SD: 0.002) in both VLF and LF] followed by $\mathrm{Coh}_{\mathrm{MAP} w m e a n}$ [VLF: 0.50 (SD: 0.001) and LF: 0.47 (SD: 0.007)] and $\mathrm{Coh}_{\text {mean }}$ [VLF: 0.47 (SD: 0.006) and LF: 0.45 (SD: 0.005)].

The variability between infants in single measurements of $\mathrm{Coh}_{\text {mean }}$ was 0.06 (Table 2), while the variability in $\mathrm{Coh}_{\text {mean }}$ within infants was 0.1 . This means that the imprecision of a measurement of coherence from a single 10-min measurement exceeded the variability in grand mean coherence between infants.

\subsection{Intrameasurement Adjustment for Blood Pressure Variability}

Overall, $\mathrm{Coh}_{\text {mean }}$ had the lowest $\mathrm{SE}$ of estimated Coherence $_{\mathrm{ST}}$. Coh $_{\mathrm{MAPwmean}}$ had a slightly higher SE of estimated Coherence ${ }_{\mathrm{ST}}$, while $\mathrm{Coh}_{\mathrm{MAPmax}}$ had the highest. The

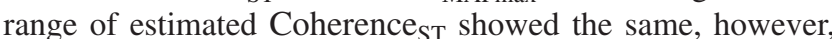
less pronounced, tendency (Table 3). Figure 3 illustrates this tendency on infant level. These observations are combined in the $P$ values of a significant difference in Coherence $_{\mathrm{ST}}$ among infants (Table 3), where $\mathrm{Coh}_{\text {mean }}$ turned out with the most significant difference. $\mathrm{Coh}_{\mathrm{MAPwm}}$ an was only able to distinguish among infants significantly in the LF-band, whereas no significant differences among infants were seen for Coh $_{\text {MAPmax }}$.

\subsection{Intermeasurement Adjustment for Blood Pressure Variability}

We found a positive but insignificant correlation between each infant's mean variability in MAP and estimates of Coherence $_{\mathrm{ST}}$ based on $\mathrm{Coh}_{\text {mean }}$ (VLF: $r^{2}=0.07, P=0.2$; LF: $\left.r^{2}=0.001, P=0.9\right)$. However, residual plots of estimated Coherence $_{\mathrm{ST}}$ (model 1) against mean $\mathrm{PSD}_{\mathrm{MAP}}$ showed a pattern of decreasing residuals with increasing mean $\mathrm{PSD}_{\mathrm{MAP}}$ (data not shown). We therefore weighted the estimated 
Hahn et al.: Precision of coherence analysis to detect cerebral autoregulation by near-infrared spectroscopy...

Table 2 Grand mean of each infant's average coherence, threshold of coherence, and standardized coherence (Coherence ${ }_{\mathrm{ST}}$ ).

\begin{tabular}{|c|c|c|c|c|c|c|c|}
\hline \multirow[b]{2}{*}{ Band } & \multirow{2}{*}{$\begin{array}{l}\text { Coherence } \\
\text { measurement }\end{array}$} & \multirow[b]{2}{*}{$N$} & \multicolumn{2}{|c|}{ Coherence } & \multirow{2}{*}{$\begin{array}{c}\text { Threshold of coherence } \\
\text { Mean }\end{array}$} & \multicolumn{2}{|c|}{ Standardized coherence } \\
\hline & & & Mean & SD & & Mean & SD \\
\hline \multirow{3}{*}{ VLF } & $\mathrm{Coh}_{\text {mean }}$ & 22 & 0.45 & 0.062 & 0.47 & -0.03 & 0.062 \\
\hline & Coh $_{\text {MAPwmean }}$ & 22 & 0.49 & 0.065 & 0.50 & -0.01 & 0.065 \\
\hline & $\mathrm{Coh}_{\text {MAPmax }}$ & 22 & 0.51 & 0.090 & 0.80 & -0.29 & 0.090 \\
\hline \multirow{3}{*}{ LF } & $\mathrm{coh}_{\text {mean }}$ & 22 & 0.44 & 0.056 & 0.45 & -0.01 & 0.056 \\
\hline & Coh $_{\text {MAPwmean }}$ & 22 & 0.46 & 0.067 & 0.47 & -0.00 & 0.067 \\
\hline & $\mathrm{Coh}_{\text {MAPmax }}$ & 22 & 0.54 & 0.120 & 0.80 & -0.26 & 0.121 \\
\hline
\end{tabular}

$\mathrm{Coh}_{\text {mean }}$ with mean $\mathrm{PSD}_{\mathrm{MAP}}$ in each measurement (intermeasurement $\mathrm{ABP}$ weighting). This weighting resulted in a wider

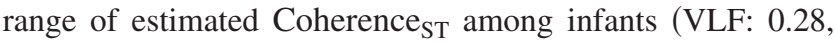
LF: 0.26) accompanied by almost no change in the SE (VLF: 0.03 , LF: 0.04). Consequently, the $P$ values were diminished (i.e., discrimination among infants increased). In VLF the $P$ value changed from 0.0013 to 0.0007 and in LF from 0.0012 to 0.0001$)$.

\subsection{Level of Coherence ${ }_{S T}$}

Because $\mathrm{Coh}_{\text {mean }}$ and intermeasurement adjustment for blood pressure variability performed best in terms of precision, we used these conditions in model 1 to estimate each infant's overall level of Coherence $_{\mathrm{ST}}$ in both frequency bands. Indication of imperfect cerebral autoregulation [i.e., Coherence $_{\mathrm{ST}} \geqslant 0$, was found in $41 \%(n=9)$ and $50 \%(n$ $=11$ ) of the infants for VLF and LF, respectively]. There was no significant difference in GA, postnatal age, or $\mathrm{P}_{\mathrm{a}} \mathrm{CO}_{2}$ between infants with an imperfect $\left(\right.$ Coherence $\left._{\mathrm{ST}} \geqslant 0\right)$ and infants with an effective autoregulation (Coherence $\left.{ }_{\mathrm{ST}}<0\right)$. Two infants received treatment with Dopamine, two infants had mechanical ventilation, two infants developed IVH grade
3-4, and two infants died in the first month of life (Table 1). None of these parameters differed significantly between the two groups.

\subsection{Statistical Power}

We estimated statistical power by the percentage among 1000 simulated data sets, where the null hypothesis (i.e., no significant difference in Coherence $_{S T}$ among infants) was rejected and plotted power against number of measurements for the three coherence measurements in VLF and LF (Fig. 4). The increase of statistical power was highest for $\mathrm{Coh}_{\text {mean }}$ with the line approaching a power of $80 \%$ by $22(3.7 \mathrm{~h})$ and $8(1.3 \mathrm{~h})$ measurements, for VLF and LF, respectively. The line for $\mathrm{Coh}_{\mathrm{MAPwmean}}$ had a much smaller slope and only reached a power of approximately $35 \%$ and $60 \%$ when simulating 35 measurements $(5.8 \mathrm{~h})$, for VLF and LF, respectively. For $\mathrm{Coh}_{\text {MAPmax }}$ the simulation study showed no change in power with an increasing number of measurements.

\subsection{Time Trend}

A time trend ( $P$ value $\leqslant 0.05$ in model 2$)$ was only demonstrated for $\mathrm{Coh}_{\text {mean }}$ in the VLF-range (data not shown). No

Table 3 Effect of adjusting for ABP variability within each measurement.

\begin{tabular}{|c|c|c|c|c|c|}
\hline \multirow[b]{2}{*}{ Band } & \multirow{2}{*}{$\begin{array}{l}\text { Coherence } \\
\text { measurement }\end{array}$} & \multicolumn{2}{|c|}{ Estimated standardized coherence } & \multirow[b]{2}{*}{ SE of estimate } & \multirow[b]{2}{*}{$p$-value } \\
\hline & & range & $(\min -\max )$ & & \\
\hline \multirow{3}{*}{ VLF } & $\mathrm{Coh}_{\text {mean }}$ & 0.214 & $(-0.110-0.104)$ & 0.04 & $0.0013^{a}$ \\
\hline & Coh $_{\text {MAPwmean }}$ & 0.220 & $(-0.132-0.088)$ & 0.05 & 0.0865 \\
\hline & Coh $_{\text {MAPmax }}$ & 0.376 & $(-0.440-0.064)$ & 0.09 & 0.5050 \\
\hline \multirow{3}{*}{ LF } & $\mathrm{Coh}_{\text {mean }}$ & 0.221 & $(-0.081-0.140)$ & 0.04 & $0.0012^{a}$ \\
\hline & Coh $_{\text {MAPwmean }}$ & 0.264 & $(-0.090-0.175)$ & 0.05 & $0.0122^{a}$ \\
\hline & Coh $_{\text {MAPmax }}$ & 0.495 & $(-0.478-0.017)$ & 0.12 & 0.5512 \\
\hline
\end{tabular}


Hahn et al.: Precision of coherence analysis to detect cerebral autoregulation by near-infrared spectroscopy...

Infant number [No. of measurements]

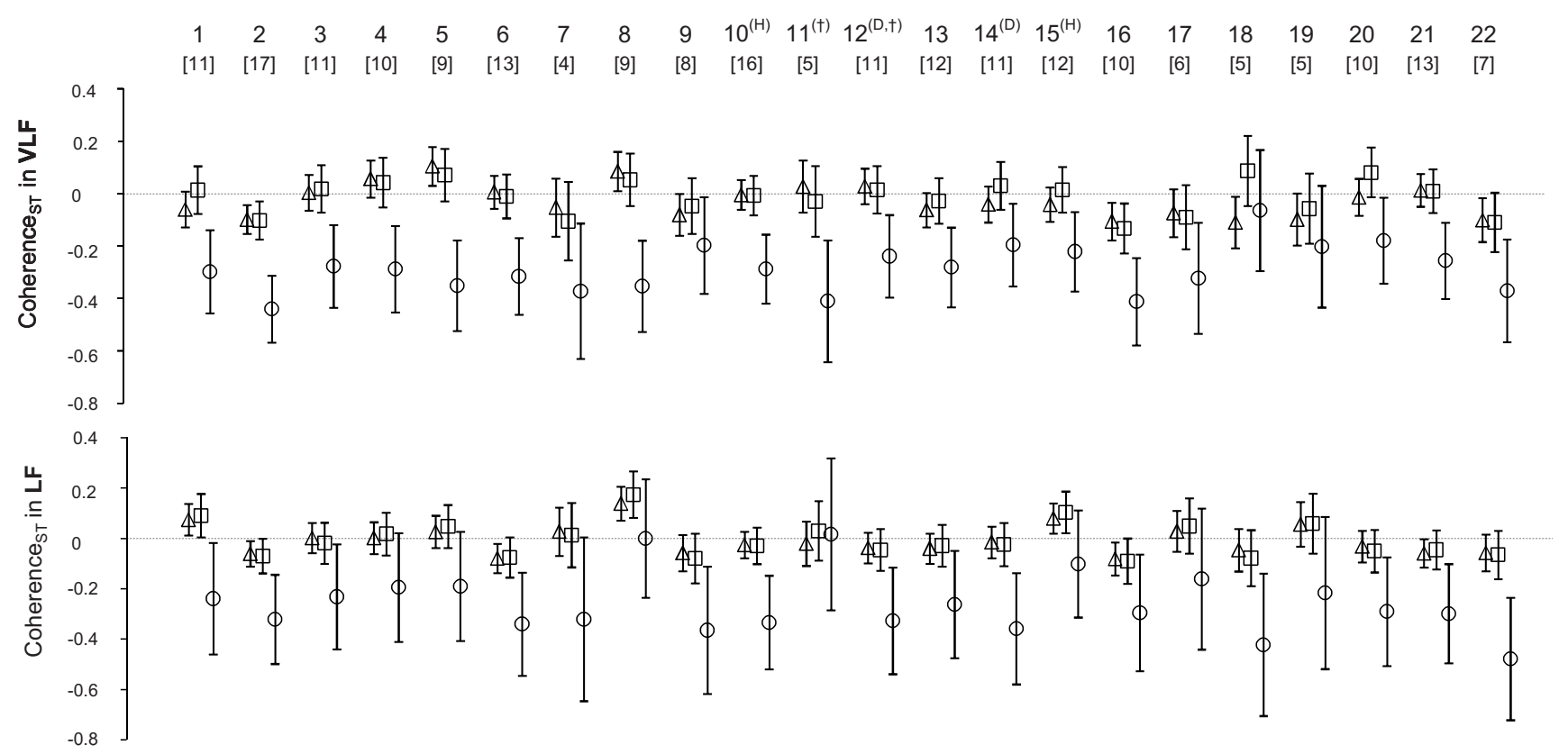

Fig. 3 Estimated Coherence ${ }_{S T}$ and number of measurements (in square brackets) in each infant illustrating the effect of intrameasurement adjustment for ABP variability in VLF and LF. Mean coherence $\left(\mathrm{Coh}_{\text {mean }}\right)[\triangle]$, intrameasurement ABP weighted mean coherence $\left(\mathrm{Coh}_{\text {MAPwmean }}\right)[\square]$, and coherence at the frequency with maximum $\mathrm{PSD}_{\text {MAP }}\left(\mathrm{Coh}_{\text {MAPmax }}\right)[\mathrm{O}$ ] are shown. Error bars represent $1.96 \times \mathrm{SE}$ of the estimate. Infants treated with Dopamine during the measurement are marked with (D). Infants who developed IVH grade 3-4 are marked with $(\mathrm{H})$. Infants who died in the first month of life are marked with ${ }^{(+)}$.

significant drift was found in the LF range, where statistical power was almost three times bigger. Consequently, our results do not provide compelling evidence of a drift in coherence over time.

\subsection{Frequency Dependency}

We found no evident concordance between estimated Coherence $_{\mathrm{ST}}$ in VLF and LF (estimates are adjusted for intermeasurement variability in ABP) (Fig. 5). Only 12 infants had concordant results when classified according to Coherence $_{\mathrm{ST}}<0$ or Coherence ST $_{2} \geqslant 0$. Even though the scatter plot is dominated by noise (i.e., wide confidence intervals

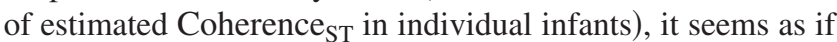
noise alone does not explain the lack of concordance between the two frequency ranges. However, the difference between estimated Coherence $_{\mathrm{ST}}$ in VLF and LF was not significant $(P=0.3$ in paired sampled T-test).

\section{Discussion}

The principal finding of this study is that the precision of coherence analysis to detect cerebral autoregulation by NIRS is improved when the magnitude of variability in ABP is taken into account. Second, it apparently requires several hours of recordings to obtain a reliable estimate of cerebral autoregulation to characterize an individual infant.

\subsection{Repeatability as a Measure of Precision}

We set out to find a method that could provide a more precise detection of cerebral autoregulation based on coherence analysis of spontaneous variations in ABP and cerebral NIRS and used repeatability as a proxy for precision. We based this
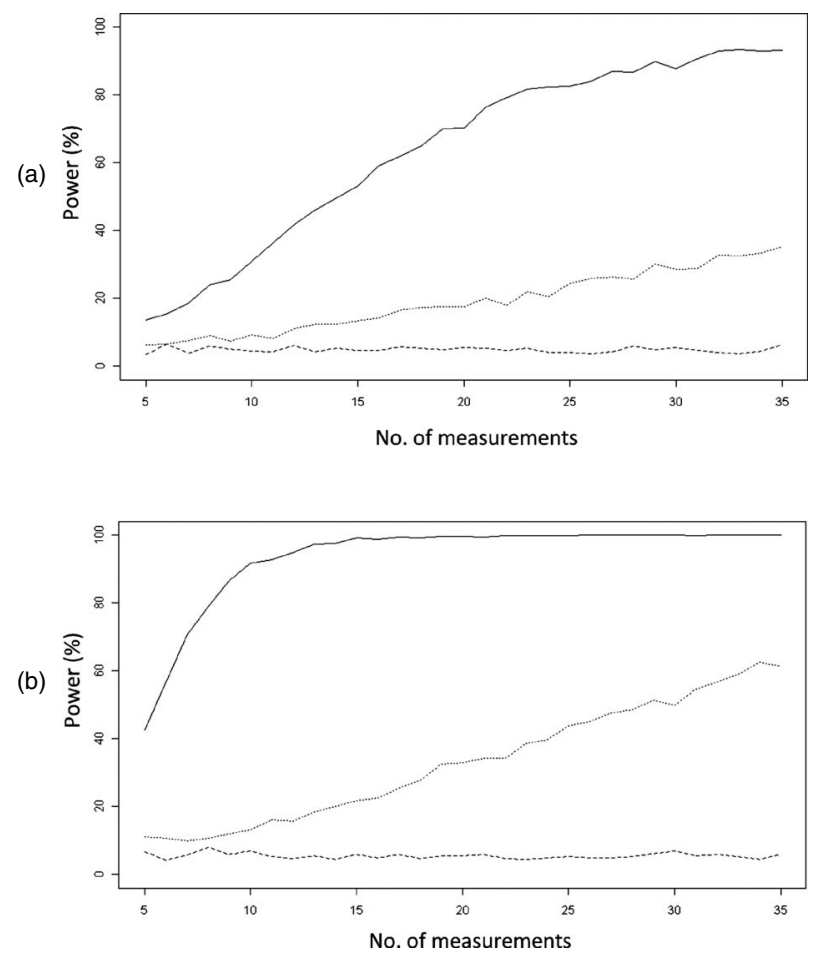

Fig. 4 Analysis of statistical power of the three coherence measurements in the (a) very low-frequency and (b) low-frequency range. Power is calculated as percentage of simulations out of 1000, where 22 infants' standardized coherence differed significantly. Mean coherence $\left(\mathrm{Coh}_{\text {mean }}\right)$ (continuous line), intrameasurement ABP weighted mean coherence ( Coh $_{\text {MAPwmean }}$ ) (dotted line), and coherence at the

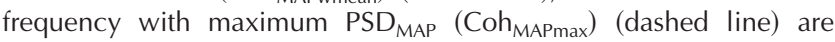
shown. 


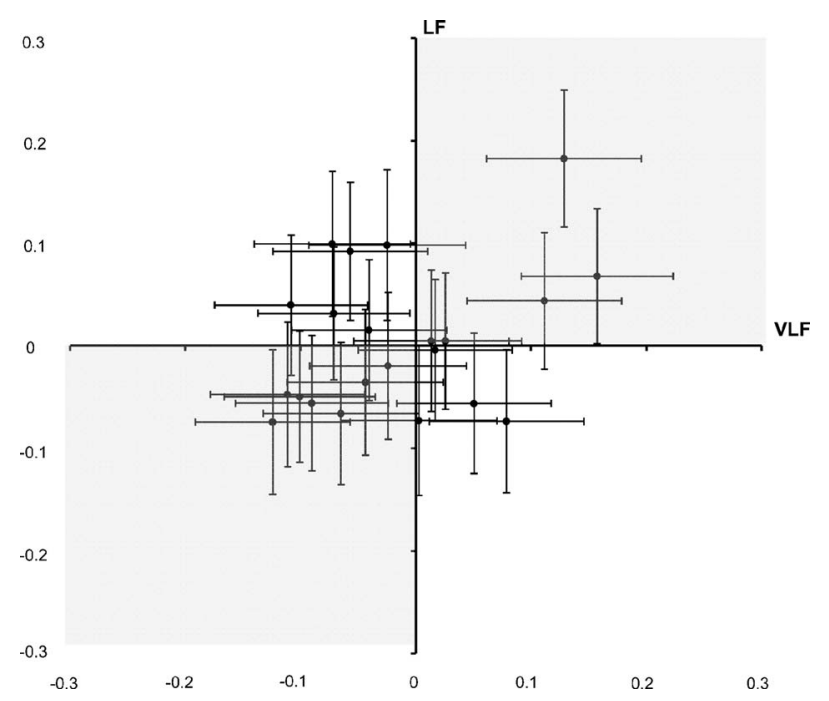

Fig. 5 Scatter plot of estimated Coherence $_{\mathrm{ST}}$ (inter-measurement adjusted for variability in ABP) in VLF and LF illustrating the amount of concordance. Error bars represent $1.96 \times$ SE of each infant's estimated Coherence $_{\mathrm{ST}}[\mathbf{0}]$. The shaded areas indicate concordance between estimated Coherence $_{\mathrm{ST}}$ in VLF and LF.

assumption on the fact that we only included clinically stable infants. Furthermore, as cerebral autoregulation reflects the overall activity of millions of vascular smooth muscle cells, it may be assumed that changes in autoregulatory capacity occur gradually. In an experimental study, autoregulation was abolished by $20 \mathrm{~min}$ of severe hypoxia and recovered over $5-7 \mathrm{~h}^{13}$ Hence, because the infants we studied were clinically stable, we assumed that cerebral autoregulation stayed reasonably stable between two successive 10-min measurements. This was in accordance with the lack of clear evidence of drift in coherence over time in individual infants.

\subsection{Adjustment for Variability in Blood Pressure}

Contrary to our expectations, we found that the mean coherence $\left(\mathrm{Coh}_{\text {mean }}\right)$ was best to discriminate among infants (i.e., had the highest precision). Thus, in other words, adjusting for variability in ABP within each measurement did not increase the signal-to-noise ratio. The same tendency was seen in our simulation study where fewer measurements (less monitoring time) were needed to discriminate among infants based on the mean coherence $\left(\mathrm{Coh}_{\text {mean }}\right)$. We ascribe the lower precision of the $\mathrm{Coh}_{\text {MAPwmean }}$ and $\mathrm{Coh}_{\text {MAPmax }}$ compared to $\mathrm{Coh}_{\text {mean }}$ to the effect of the signal-to-noise ratio in MAP. The noise is transferred to the power spectrum by the Fourier transform and gives rise to uncertainty in determining the weighting and the frequency with maximum power, respectively.

However, in line with our hypothesis, precision improved when we adjusted for the varying degree of variability in ABP between measurements in each infant (intermeasurement ABP weighting). Thus, in other words, weighting measurements with large fluctuations in $\mathrm{ABP}$ in favor of those with small actually increased the signal-to-noise ratio. Unfortunately, a power study using simulated data sets could not be conducted to explore how this weighting would affect the monitoring time because it would require simulating an ABP signal.
To our knowledge, no previous studies have investigated the influence of variability in $\mathrm{ABP}$ on estimated coherence between spontaneous fluctuations in ABP and cerebral NIRS measurements. Our results, however, are in line with previous studies of cerebral autoregulation based on transfer function analysis between Doppler measurements of cerebral blood flow velocity and spontaneous variations in $\mathrm{ABP} .^{14,15}$ It has been proposed to set a minimum variability in $\mathrm{ABP}$ for a more precise estimation of cerebral autoregulation from spontaneous fluctuations in ABP. ${ }^{5,14}$ However, this approach will result in data loss, because in practice, the variability in ABP can be quite low over a considerable length of time. It is important to use data as effectively as possible when research involves vulnerable patients, such as premature infants. Hence, our approach with weighting coherence with variability in ABP in each measurement seems relevant for research studies comparing cerebral autoregulation among vulnerable patients.

\subsection{Frequency-Dependent Functional Relationship}

Simulations to estimate the statistical power of the various signal analytical approaches showed a remarkable difference in statistical power in the two frequency ranges. Statistical power was almost three times bigger in LF compared to VLF. From a practical point of view, this indicates that a threetimes-longer recording time is required in VLF compared to LF. We speculate that this finding might be attributed to the fact that other regulatory mechanisms for cerebral hemodynamics have more influence in the VLF band (i.e., $0.003-0.4 \mathrm{~Hz}, 25-300 \mathrm{~s}$ cycle length) compared to the LF band (i.e., $0.04-0.1 \mathrm{~Hz}, 10-25$ s cycle length). This will result in a lower signal-to-noise ratio. Because $91 \%$ of our infants were breathing spontaneously, irregular fluctuations in $\mathrm{P}_{\mathrm{a}} \mathrm{CO}_{2}$ might have contributed to the lower statistical power in the VLF band.

The lack of concordance between VLF and LF (Fig. 5) implies that they might represent different aspects or time scales of cerebral autoregulation. Because the autoregulatory ability is controlled by vascular smooth muscle cells, we speculate that an injurious stimulus initially reduces the response time and then eventually destroys the ability to react to changes in blood pressure. Consequently, an infant might actually have a bad autoregulation at higher frequencies and a good one at lower frequencies (upper left quadrant on Fig. 5). However, a higher amount of physiological noise in VLF compared to LF could also contribute to the lack of concordance by falsely indicating a good autoregulation at low frequencies. Still, further analyses are needed to clarify if the two frequency bands contribute with shared or different information.

\subsection{Nature of Cerebral Autoregulation}

Our results indicated a range of levels of coherence, even in these relatively healthy preterm infants. Furthermore, the drift in individual infants was minimal. In an earlier work by Soul et al., ${ }^{8}$ who used the same technique to detect cerebral autoregulation in preterm infants, data were analyzed and interpreted differently. The authors computed a pressure-passive index (i.e., percentage of all 10-min measurements that were classified as coherent) to characterize autoregulation in each 
infant. The computation of Soul et al.'s study was based on an assumption of autoregulation being "on" or "off" according to the mean coherence being below or above the threshold of statistical significance. Our data show that single values of coherence from 10-min measurements have an imprecision that exceeds the variability among infants. Consequently, we believe that the precision of this measuring method is too low to get a meaningful estimate from a single measurement. Similarly, using the maximum value of coherence (i.e., picking a single measurement among several) to characterize each infant's level of autoregulation is not an effective nor unbiased way of data analysis. In two previous studies, this was done and the maximum coherence was associated with mortality ${ }^{7}$ and occurrence of IVH in premature infants. ${ }^{9}$

An imperfect cerebral autoregulation (i.e., coherence exceeding the limit of statistical significance) was detected in approximately one-half of our stable infants, and no correlation was found to disease (i.e., IVH, neonatal death, or use of Dopamine). The fact that an imperfect cerebral autoregulation did not correlate with disease was unexpected. However, the present study was not powered to test for this correlation. The proportion of infants with an imperfect cerebral autoregulation is in line with earlier studies using the same technique to study "dynamic" cerebral autoregulation in premature infants. ${ }^{7,9}$ Contrarily, studies using a "static" measuring approach have, in general, shown an intact cerebral autoregulation in stable premature infants and an impaired autoregulation in sick premature infants. The discrepancy between studies based on continuous cerebral NIRS and studies based on single measurements of cerebral blood flow by means of either xenon-133 clearance technique ${ }^{16,17}$ or cerebral NIRS $^{18,19}$ may be apparent. The conclusion that autoregulation is present in stable premature infants in the latter studies came from a statistically insignificant relation between arterial blood flow and cerebral blood flow. In fact, the 95\%confidence limit was $+2 \% / \mathrm{mm} \mathrm{Hg}$ (i.e., compatible with an imperfect autoregulation that would give rise to a statistically significant coherence with the techniques discussed here).

\subsection{Strength and Limitations}

\subsubsection{Appropriate threshold of significance}

Strength of our technique is that we used a measurementspecific threshold of significant coherence based on Monte Carlo simulations. Previous reports in this field using mean analysis of coherence have either used a conventional threshold of significant coherence of $0.5^{7,9,20}$ or a calculated threshold ${ }^{8}$ based on a published mathematical definition. ${ }^{21}$ However, a conventional threshold cannot be generalized because it does not account for differences in analytical conditions such as length of measurements and segments, number of harmonics in the frequency range, type of tapering window applied, or degree of overlap between segments. These differences affect the degrees of freedom of the estimated coherence and thus, the threshold of significant coherence. ${ }^{5,22} \mathrm{Be}$ sides, the published mathematical definition is only valid for point measurements. ${ }^{21}$ Our Threshold ${ }_{\mathrm{COH}}$ for $\mathrm{Coh}_{\text {mean }}$ and $\mathrm{Coh}_{\mathrm{MAPwmean}}$ happened to be close to the conventional limit of 0.5. Threshold $\mathrm{COH}$ for our point measurement $\left(\mathrm{Coh}_{\text {MAPmax }}\right)$ was almost identical to a calculated threshold of 0.78 based on our analytical conditions [i.e., sampling rate
( $2 \mathrm{~Hz}$ ), length of measurements (10 $\mathrm{min}=1200$ samples), and length of segments $(5 \mathrm{~min}=600$ samples $)]$. The fact that our Threshold $_{\mathrm{COH}}$ showed very low standard deviations indicates that it may not be necessary to run Monte Carlo simulations on all of the recorded data. A previous study ${ }^{23}$ has claimed an advantage of this method because it maintains the statistical distribution of the original data. However, we found that the threshold is only changed significantly by the way the coherence is calculated. Hence, we propose that a reliable threshold of significant coherence can be achieved by running Monte Carlo simulations on a single recording representing the analytical conditions.

\subsubsection{Oxygenation versus blood flow}

We used the oxygenation index as a surrogate measure of cerebral blood flow. This assumption is only reasonable if arterial oxygenation is stable during measurements. We kept the impact of variations in arterial oxygenation to a minimum by rejecting all data with changes in arterial saturation exceeding 5\%. The cerebrovascular transit time (i.e., the time delay in reaching equilibrium of oxygenation as response to changes in cerebral blood flow) represents another potential limitation when oxygenation index is used as a surrogate measure of cerebral blood flow. ${ }^{24}$ However, because cerebrovascular transit time in neonates is $\sim 10 \mathrm{~s},{ }^{25}$ this limitation would not affect analysis in our frequency range. The fact that cerebral oxygenation is "volume weighted" and not "flow weighted" 26 represents another problem. This is relevant if the volume of tissue is heterogeneous, as is the case with the brain. Change in mean blood flow is dominated by changes to high flow regions, whereas change in mean (vascular) oxygenation is dominated by changes in high blood volume regions. Consequently, changes in oxygenation will only truly reflect changes in blood flow if the following conditions are met: $(i)$ changes in blood flow to all parts of the volume of tissue are proportional and (ii), changes in blood flow and blood volume are proportional. These are reasonable assumptions in normal conditions and during small changes, but it is difficult to know.

\subsubsection{Other factors influencing cerebral blood flow}

Another potential limitation of our approach lies in the very concept of using coherence to estimate cerebral autoregulation. Coherence assumes linearity between ABP and cerebral blood flow (i.e., it assumes ABP to drive cerebral blood flow), thereby ignoring other potential input signals. Thus, it does not account for a possible effect of other physiological variables on cerebral blood flow (e.g., $\mathrm{P}_{\mathrm{a}} \mathrm{CO}_{2}$ ). We tried to keep this problem to a minimum by only investigating infants who were clinically stable.

\subsubsection{Statistical versus physiological significance}

Strictly speaking a coherence exceeding the threshold of statistical significance only indicates that autoregulation is not working perfectly (i.e., that the slope of cerebral blood flow versus ABP is not precisely zero). The quantitative assessment of this slope (i.e., the actual value of autoregulation) requires estimation of the operational gain (i.e., magnitude of change in cerebral oxygenation per change in ABP). Because auto- 
regulation is always imperfect to some degree, a statistical significant coherence could correspond to an imperfect but not impaired cerebral autoregulation.

\section{Conclusion}

A reliable detection of cerebral autoregulation by means of analysis of coherence between cerebral NIRS and spontaneous fluctuations in ABP requires several hours of monitoring. The precision can be improved by adjusting for the varying degree of blood pressure variability between repeated measurements. These findings are relevant for research studies comparing cerebral autoregulation among vulnerable patients.

\section{Acknowledgments}

We thank the Ludvig and Sara Elsass Foundation and the University of Copenhagen, Denmark, for funding the study. We also thank the participating infants and their parents for contributing to this study. Finally, we thank the staff of the Neonatal Department and the Medical Engineering Department at Rigshospitalet for practical and technical assistance, respectively.

\section{References}

1. J. E. Brazy, D. V. Lewis, M. H. Mitnick, and V. Jobsis, "Noninvasive monitoring of cerebral oxygenation in preterm infants: preliminary observations," Pediatrics 75(2), 217-225 (1985)

2. G. Greisen, "Autoregulation of cerebral blood flow," NeoReviews 8(1), 22-31 (2007)

3. J. J. Volpe, "Neurobiology of periventricular leukomalacia in the premature infant," Pediatr. Res. 50(5), 553-562 (2001).

4. C. A. Giller and D. G. Iacopino, "Use of middle cerebral velocity and blood pressure for the analysis of cerebral autoregulation at various frequencies: the coherence index," Neurol. Res. 19(6), 634-640 (1997).

5. R. B. Panerai, "Cerebral autoregulation: from models to clinical applications," Cardiovasc. Eng. 8(1), 42-59 (2008).

6. E. G. Ramos, D. M. Simpson, R. B. Panerai, J. Nadal, J. M. Lopes, and D. H. Evans, "Objective selection of signals for assessment of cerebral blood flow autoregulation in neonates," Physiol. Meas 27(1), 35-49 (2006).

7. F. Y. Wong, T. S. Leung, T. Austin, M. Wilkinson, J. H. Meek, J. S Wyatt, and A. M. Walker, "Impaired autoregulation in preterm infants identified by using spatially resolved spectroscopy," Pediatrics 121(3), e604-e611 (2008).

8. J. S. Soul, P. E. Hammer, M. Tsuji, J. P. Saul, H. Bassan, C. Limperopoulos, D. N. Disalvo, M. Moore, P. Akins, S. Ringer, J. J. Volpe, F. Trachtenberg, and A. J. du Plessis, "Fluctuating pressure-passivity is common in the cerebral circulation of sick premature infants," Pediatr. Res. 61(4), 467-473 (2007).

9. M. Tsuji, J. P. Saul, P. A. du, E. Eichenwald, J. Sobh, R. Crocker, and J. J. Volpe, "Cerebral intravascular oxygenation correlates with mean arterial pressure in critically ill premature infants," Pediatrics 106(4), 625-632 (2000).

10. M. Tsuji, A. duPlessis, G. Taylor, R. Crocker, and J. J. Volpe, "Near infrared spectroscopy detects cerebral ischemia during hypotension in piglets," Pediatr. Res. 44(4), 591-595 (1998).

11. J. S. Soul, G. A. Taylor, D. Wypij, A. J. Duplessis, and J. J. Volpe, "Noninvasive detection of changes in cerebral blood flow by nearinfrared spectroscopy in a piglet model of hydrocephalus," Pediatr. Res. 48(4), 445-449 (2000).

12. A. Pryds, J. Tonnesen, O. Pryds, G. M. Knudsen, and G. Greisen, "Cerebral pressure autoregulation and vasoreactivity in the newborn rat," Pediatr. Res. 57(2), 294-298 (2005).

13. A. Tweed, J. Cote, H. Lou, G. Gregory, and J. Wade, "Impairment of cerebral blood flow autoregulation in the newborn lamb by hypoxia," Pediatr. Res. 20(6), 516-519 (1986).

14. J. Liu, D. M. Simpson, and R. Allen, "High spontaneous fluctuation in arterial blood pressure improves the assessment of cerebral autoregulation," Physiol. Meas 26(5), 725-741 (2005).

15. D. M. Simpson, R. B. Panerai, E. G. Ramos, J. M. Lopes, M. N. Marinatto, J. Nadal, and D. H. Evans, "Assessing blood flow control through a bootstrap method," IEEE Trans. Biomed. Eng. 51(7), 1284-1286 (2004)

16. D. Jayasinghe, A. B. Gill, and M. I. Levene, "CBF reactivity in hypotensive and normotensive preterm infants," Pediatr. Res. 54(6), 848-853 (2003).

17. O. Pryds, G. Greisen, H. Lou, and B. Friis-Hansen, "Heterogeneity of cerebral vasoreactivity in preterm infants supported by mechanical ventilation," J. Pediatr. 115(4), 638-645 (1989).

18. L. Tyszczuk, J. Meek, C. Elwell, and J. S. Wyatt, "Cerebral blood flow is independent of mean arterial blood pressure in preterm infants undergoing intensive care," Pediatrics 102(2 Pt 1), 337-341 (1998).

19. M. J. Munro, A. M. Walker, and C. P. Barfield, "Hypotensive extremely low birth weight infants have reduced cerebral blood flow," Pediatrics 114(6), 1591-1596 (2004).

20. H. Bassan, K. Gauvreau, J. W. Newburger, M. Tsuji, C. Limperopoulos, J. S. Soul, G. Walter, P. C. Laussen, R. A. Jonas, and A. J. du Plessis, "Identification of pressure passive cerebral perfusion and its mediators after infant cardiac surgery," Pediatr. Res. 57(1), 35-41 (2005).

21. J. A. Taylor, D. L. Carr, C. W. Myers, and D. L. Eckberg, "Mechanisms underlying very-low-frequency RR-interval oscillations in humans," Circulation 98(6), 547-555 (1998).

22. R. Bortel and P. Sovka, "Approximation of statistical distribution of magnitude squared coherence estimated with segment overlapping," Signal Process. 87(5), 1100-1117 (2007).

23. R. B. Panerai, P. J. Eames, and J. F. Potter, "Multiple coherence of cerebral blood flow velocity in humans," Am. J. Physiol. Heart Circ. Physiol. 291(1), H251-H259 (2006).

24. F. Y. Wong, M. Nakamura, T. Alexiou, V. Brodecky, and A. M. Walker, "Tissue oxygenation index measured using spatially resolved spectroscopy correlates with changes in cerebral blood flow in newborn lambs," Intensive Care Med. 35(8), 1464-1470 (2009).

25. C. E. Elwell, M. Cope, and D. T. Delpy, "An analytical method for determining cerebrovascular transit time using near infrared spectroscopy," Adv. Exp. Med. Biol. 428, 561-568 (1997).

26. M. Smith and C. Elwell, "Near-infrared spectroscopy: shedding light on the injured brain," Anesth. Analg. 108(4), 1055-1057 (2009). 